\title{
SYNTHESIS OF SYMMETRICAL
}

\section{TETRAARYLTETRA[2,3]NAPHTHO-}

\author{
PORPHYRINS
}

\section{SUPPORTING INFORMATION}

Olga S. Finikova, ${ }^{\mathrm{a}}$ Sergei E. Aleshchenkov, ${ }^{\mathrm{b}}$ Raymond P. Briñas, ${ }^{\mathrm{a}}$ Andrei $V$. Cheprakov, ${ }^{\mathrm{b} *}$ Patrick J. Carroll ${ }^{\mathrm{c}}$ and Sergei A. Vinogradov ${ }^{\mathrm{a} *}$

${ }^{a}$ Department of Biochemistry and Biophysics, University of Pennsylvania, Philadelphia, PA 19104; ${ }^{b}$ Department of Chemistry, Moscow State University, Moscow 119899, Russia; ${ }^{c}$ Department of Chemistry, Crystallographic Laboratory, University of Pennsylvania, Philadelphia, PA 19104.

* To whom correspondence should be addressed. Phone: (215)-898-6382, FAX: (215)573-3787, E-mail: vinograd@mail.med.upenn.edu 


\section{Table of Contents}

Materials and Methods___S3

X-ray Structure Determination___ S3

Normal-Coordinate Structural Decomposition (NSD) Analysis___ S6

References and Notes___ S7

NMR spectra__ S8 


\section{Materials and Methods}

All solvents were purified according to the standard procedures. Selecto silica gel or neutral aluminum oxide (Brockmann I, 150 mesh) was used for column chromatography. All starting materials and reagents were obtained from standard commercial suppliers. Analytical TCL's were run on commercial Riedel-de Haën plates coated with silica gel $60 \mathrm{~F}_{264}(0.2-\mathrm{mm}$ thickness).

Static fluorescence and phosphorescence measurements were performed on SPEX Fluorolog-2 spectrofluorometer (Jobin-Yvon Horiba, Inc.), equipped with an infra-red enhanced R2658P PMT (Hamamatsu). Phosphorescence lifetimes were measured using an in-house constructed frequency domain phosphorometer. ${ }^{1}$ Solutions for phosphorescence measurements were purged with Ar. The absorbances of the samples at the wavelengths used for excitation were kept below 0.05 OD. The quantum yields were obtained by computing the integrals of the corrected emission spectra and referencing them to the fluorescence quantum yield of ZnTPP in deoxygenated benzene $\left(\phi_{\text {fluor }}=0.033\right) .^{2}$ The spectra were normalized by the optical density of the samples at the excitation wavelengths, relative photon intensity of the source and quantum efficiency of the detector throughout the emission range.

Phosphorescence oxygen quenching constants $\left(\mathrm{K}_{\mathrm{q}}\right)$ were determined as described previously. ${ }^{3}$

\section{X-ray Structure Determination}

Compound 12f, $\mathrm{C}_{128} \mathrm{H}_{134} \mathrm{~N}_{4} \mathrm{O}_{25} \mathrm{Pd}$, crystallizes in the orthorhombic space group Pna2 $_{1}$ (systematic absences $\mathrm{h} 01: \mathrm{h}=$ odd and $0 \mathrm{kl}: \mathrm{k}+\mathrm{l}=\mathrm{odd}$ ) with $\mathrm{a}=34.324(2) \AA$, $\mathrm{b}=18.0281(8) \AA, \mathrm{c}=18.0481(8) \AA, \mathrm{V}=11168.1(9) \AA^{3}, \mathrm{Z}=4$ and $\mathrm{d}_{\text {calc }}=1.329 \mathrm{~g} / \mathrm{cm}^{3}$. X-ray intensity data were collected on a Rigaku Mercury CCD area detector employing graphite-monochromated Mo- $\mathrm{K}_{\alpha}$ radiation $(\lambda=0.71069 \AA)$ at a temperature of $143 \mathrm{~K}$. Preliminary indexing was performed from a series of twelve $0.5^{\circ}$ rotation images with exposures of $30 \mathrm{~s}$. A total of 990 rotation images were collected with a crystal to detector distance of $50 \mathrm{~mm}$, a $2 \theta$ swing angle of $-15^{\circ}$, rotation widths of $0.5^{\circ}$ and exposures of 75 s: $\operatorname{scan}$ No. 1 was a $\phi$-scan from $165^{\circ}$ to $495^{\circ}$ at $\omega=10^{\circ}$ and $\chi=20^{\circ}$; scan No. 2 was a $\phi$-scan from $123.75^{\circ}$ to $288.75^{\circ}$ at $\omega=0^{\circ}$ and $\chi=-30^{\circ}$. Rotation images were processed using CrystalClear, ${ }^{4}$ producing a listing of unaveraged $\mathrm{F}^{2}$ and $\sigma\left(\mathrm{F}^{2}\right)$ values which were then passed to the CrystalStructure ${ }^{5}$ program package for further processing and structure solution on a Dell Pentium III computer. A total of 76714 reflections were measured over the ranges $5.1^{\circ} \leq 2 \theta \leq 50.1^{\circ},-40 \leq \mathrm{h} \leq 38,-21 \leq \mathrm{k} \leq 21,-20 \leq 1 \leq 21$ yielding 18088 unique reflections $\left(\mathrm{R}_{\text {int }}=0.0526\right)$. The intensity data were corrected for Lorentz and polarization effects and for absorption using REQAB (minimum and maximum transmission $0.965,1.000$ ).

The structure was solved by direct methods $\left(\operatorname{SIR} 97^{6}\right)$. The asymmetric unit includes a molecule of diethyl ether solvent that was refined as a rigid group. There were several areas of large thermal motion/disorder in the porphyrin alkyl chains. A partial occupancy disorder molecule was devised for the C121-C124 moiety utilizing a restrained bond length refinement; there were several other areas (C64-C67, C69-C72, C90-C93) that displayed large anisotropic thermal motion, for which reasonable disorder 
models could not be devised. Restrained bond length refinement was used in the C69$\mathrm{C} 72$ chain. There is a short $\mathrm{H}-\mathrm{H}$ contact $(\mathrm{H} 121 \mathrm{a}-\mathrm{H} 125 \mathrm{a}, 1.79 \AA)$ which is a reflection of the difficulty of modeling the disordered solvent and alkyl chain C121-C124.

Refinement was by full-matrix least squares based on $\mathrm{F}^{2}$ using SHELXL-97 ${ }^{7}$. All reflections were used during refinement $\left(\mathrm{F}^{2}\right.$, s that were experimentally negative were replaced by $\left.\mathrm{F}^{2}=0\right)$. The weighting scheme used was $\mathrm{w}=1 /\left[\sigma^{2}\left(\mathrm{~F}_{\mathrm{o}}^{2}\right)+0.1685 \mathrm{P}^{2}+\right.$ $127.8155 \mathrm{P}]$ where $\mathrm{P}=\left(\mathrm{F}_{\mathrm{o}}^{2}+2 \mathrm{~F}_{\mathrm{c}}^{2}\right) / 3$. Non-hydrogen atoms were refined anisotropically and hydrogen atoms were included as constant contributions to the structure factors and were not refined. Refinement converged to $\mathrm{R}_{1}=0.1090$ and $\mathrm{wR}_{2}=0.3191$ for 15819 reflections for which $\mathrm{F}>4 \sigma(\mathrm{F})$ and $\mathrm{R}_{1}=0.1231, \mathrm{wR}_{2}=0.3322$ and $\mathrm{GOF}=1.070$ for all 18088 unique, non-zero reflections and 1424 variables. ${ }^{8}$ The maximum $\Delta / \sigma$ in the final cycle of least squares was 0.032 and the two most prominent peaks in the final difference Fourier were +2.223 and $-1.208 \mathrm{e} / \AA^{3}$.

Table 1 lists cell information, data collection parameters, and refinement data. Figure 1 shows the atom numbering scheme and Figure 2 is an ORTEP ${ }^{9}$ representation of the molecule with $30 \%$ probability thermal ellipsoids displayed.

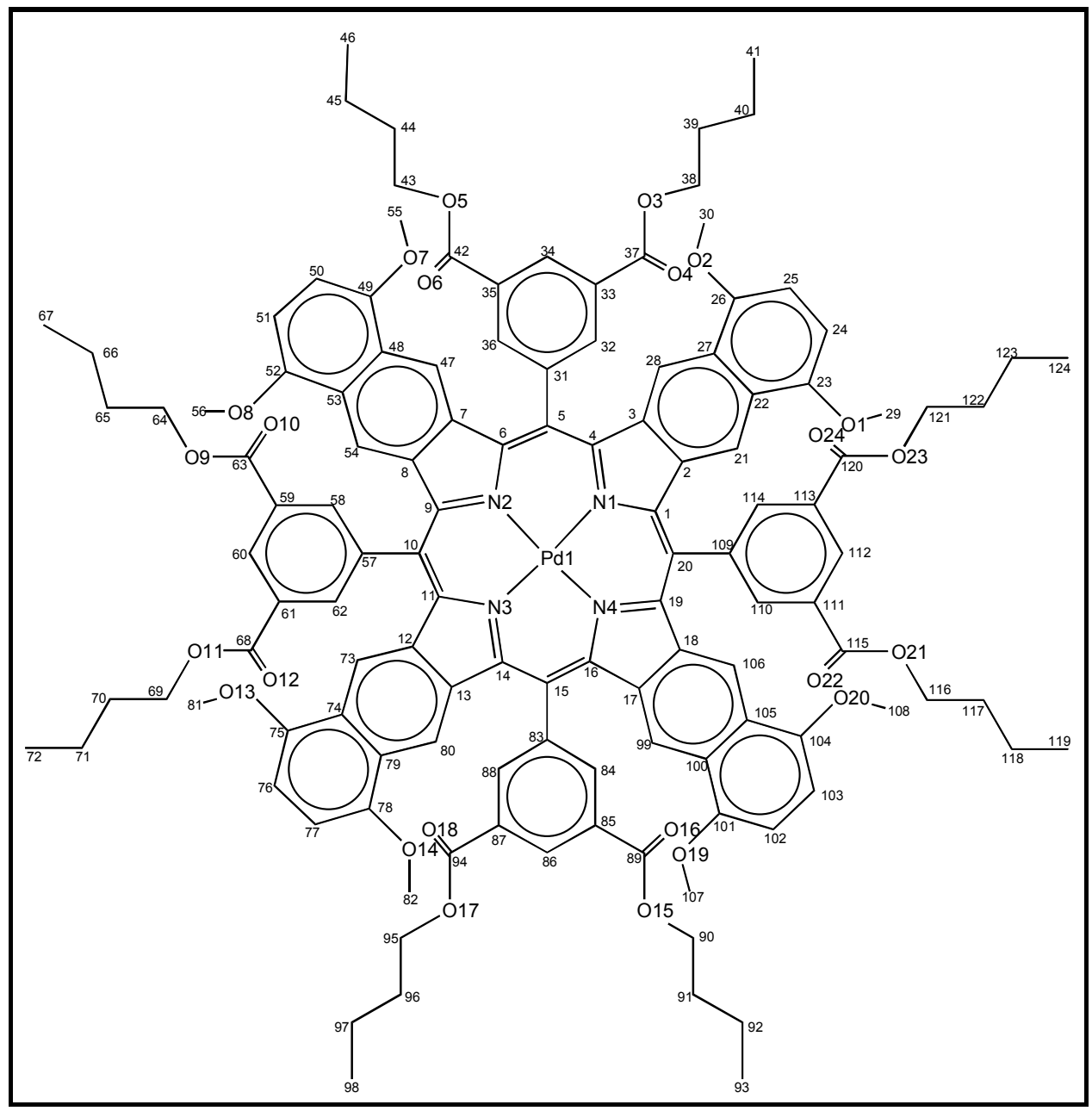

Figure 1. Atom numbering scheme. 


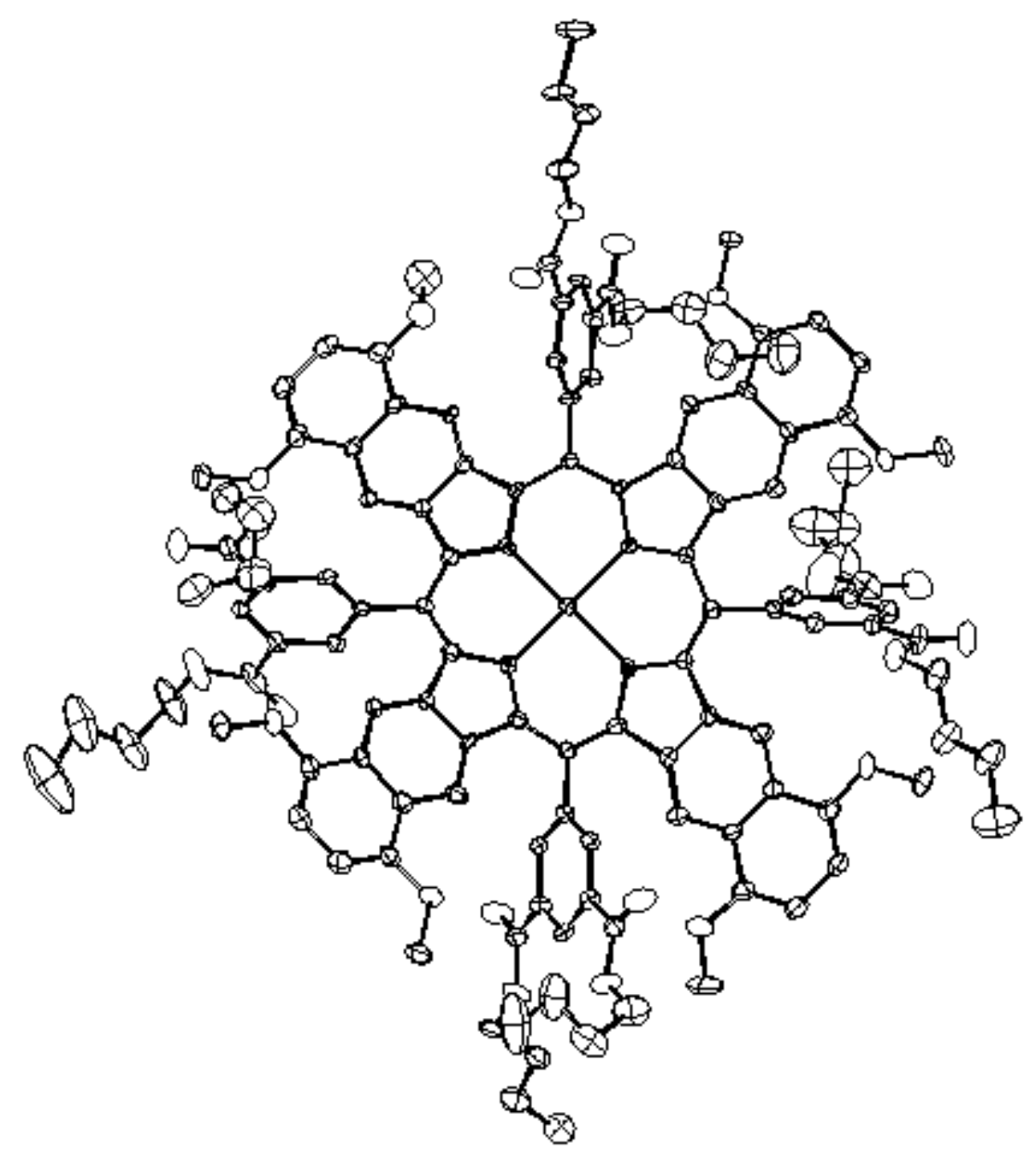

Figure 2. ORTEP drawing of the molecule with $30 \%$ probability thermal ellipsoids.

Table 1. Summary of Structure Determination for Compound Pd-12f

Formula:

Formula weight:

Crystal class:

Space group:

Z

Cell constants:

a

b

c

V

$\mu$

$\mathrm{C}_{128} \mathrm{H}_{134} \mathrm{~N}_{4} \mathrm{O}_{25} \mathrm{Pd}$

2234.79

orthorhombic

Pna2 1 (\#33)

4

$34.324(2) \AA$

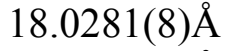

$18.0481(8) \AA$

$11168.1(9) \AA^{3}$

$2.44 \mathrm{~cm}^{-1}$ 
crystal size, $\mathrm{mm}$

$\mathrm{D}_{\text {calc }}$

$\mathrm{F}(000)$

Radiation:

$2 \theta$ range

hkl collected:

No. reflections measured:

No. unique reflections:

No. observed reflections

No. reflections used in refinement

No. parameters

$R$ indices $(F>4 \sigma)$

$\mathrm{R}$ indices (all data)

GOF:

Final Difference Peaks, e $/ \AA^{3}$
$0.25 \times 0.10 \times 0.08$

$1.329 \mathrm{~g} / \mathrm{cm}^{3}$

4704

Mo- $\mathrm{K}_{\alpha}(\lambda=0.71073 \AA)$

$5.1-50.1^{\circ}$

$-40 \leq \mathrm{h} \leq 38 ;-21 \leq \mathrm{k} \leq 21$;

$-20 \leq 1 \leq 21$

76714

$18088\left(\mathrm{R}_{\text {int }}=0.0526\right)$

$15819(\mathrm{~F}>4 \sigma)$

18088

1424

$\mathrm{R}_{1}=0.1090$

$\mathrm{wR}_{2}=0.3191$

$\mathrm{R}_{1}=0.1231$

$\mathrm{wR}_{2}=0.3322$

1.070

$+2.223,-1.208$

\section{Normal Coordinate Structural Decomposition (NSD) analysis}

NSD analysis of the structures of $\mathbf{P d - 1 2} \mathbf{b}^{10}$ and $\mathbf{P d - 1 2 f}$ was accomplished using the web-based NSD program. ${ }^{11}$ The results of the complete NSD decomposition are given below.

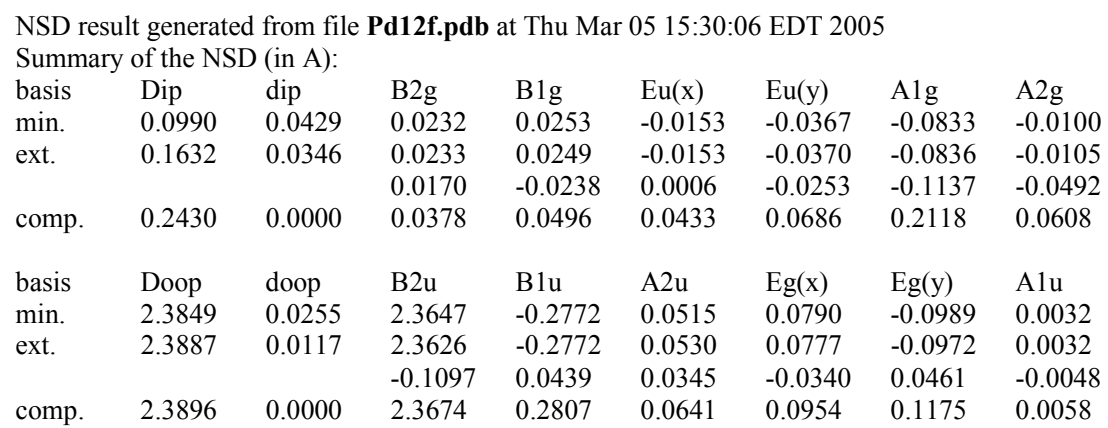




\section{References and Notes}

(1) Vinogradov, S. A.; Fernandez-Seara, M. A.; Dugan, B. W.; Wilson, D. F. Rev. Sci. Instrum. 2001, 72, 3396.

(2) Quimby, D. J.; Longo, F. R. J. Am. Chem. Soc. 1975, 97, 5111.

(3) Rozhkov, V.V.; Wilson, D.F.; Vinogradov, S.A. Macromolecules 2002, 35, 1991.

(4) CrystalClear: Rigaku Corporation, 1999.

(5) CrystalStructure: Crystal Structure Analysis Package, Rigaku Corp. Rigaku/MSC (2002).

(6) SIR97: Altomare, A., M. Burla, M. Camalli, G. Cascarano, C. Giacovazzo, A. Guagliardi, A. Moliterni, G. Polidori \& R. Spagna (1999). J. Appl. Cryst., 32, $115-$ 119.

(7) SHELXL-97: Program for the Refinement of Crystal Structures, Sheldrick, G.M., 1997, University of Göttingen, Germany.

(8) $\quad \mathrm{R}_{1}={ }_{-}|| \mathrm{F}_{\mathrm{O}}|-| \mathrm{F}_{\mathrm{C}}\left|/ /_{-}\right| \mathrm{F}_{\mathrm{O}} \mid$

$\mathrm{wR}_{2}=\left\{-\mathrm{w}\left(\mathrm{F}_{\mathrm{o}}^{2}-\mathrm{F}_{\mathrm{c}}^{2}\right)^{2} /-\mathrm{w}\left(\mathrm{F}_{\mathrm{o}}^{2}\right)^{2}\right\}^{1 / 2}$

$\mathrm{GOF}=\left\{\_\mathrm{w}\left(\mathrm{F}_{\mathrm{o}}^{2}-\mathrm{F}_{\mathrm{c}}^{2}\right)^{2} /(\mathrm{n}-\mathrm{p})\right\}^{1 / 2}$

where $\mathrm{n}=$ the number of reflections and $\mathrm{p}=$ the number of parameters refined.

(9) “ORTEP-II: A Fortran Thermal Ellipsoid Plot Program for Crystal Structure Illustrations.” C.K. Johnson, 1976, ORNL-5138.

(10) Finikova, O. S.; Cheprakov, A. V.; Carroll, P. J.; Vinogradov, S. A. J. Org. Chem. 2003, 68, 7517.

(11) http://jasheln.unm.edu/ NSD references: (a) W. Jentzen, X.-Z. Song, J. A. Shelnutt, J. Phys.Chem. B 1997, 101, 1684-1699; (b) W. Jentzen, J. G. Ma, J. A. Shelnutt, Biophys. J. 1998, 74, 753-763; For reviews see: (c) J. Shelnutt, Molecular simulations and normal-coordinate structural analysis of porphyrins and heme proteins, Ch. 50 in The Porphyrin Handbook, K. M. Kadish, K. M. Smith, R. Guilard, Eds., Academic Press, 2000; (d) J. A. Shelnutt, J. Porph. Phthal. 2001, 5, 300. 


\section{NMR spectra}

\section{Starting materials}




\section{Pyrrol-ester 4} 1H NMR

59

USER: -2 DATE: Oct 52002 


\section{pyrrol-ester 4} 13C NMR

W.w.

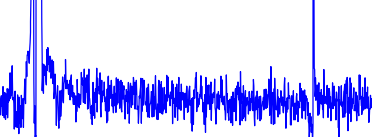

SIO

LB: 0.0
PTS 1d: 8192 


\section{6a-dication}

CDCl3 - TFA 


\section{6b-dication} CDCl3 - TFA

\begin{abstract}
F2: 499.855
\end{abstract}
SW1: 20000

PW: 5.0 usec

OF1: 2491.5

NA: 50

LB: 0.0 PTS1d: 32768

WinNuts - \$(4-MeO2C)Ph4TOP_dc_tfa 


\section{Pd-6a \\ CDCl3}

18.70

NA: 8

\begin{abstract}
F2: 499.842
\end{abstract}
EX: s $\angle$ pul 


\section{Pd-6b \\ CDCl3}

$\$ 14$

16.85

2.94

4.00

6

5

3

2

DPM

STANDARD PROTON PARAMETERS

F1: 499.855

F2: 499.855

SW1: 20000

PW: 1.5 usec

OF1: 2491.1

USER: -- DATE: Oct 82002

EX: s2pul

PD: 0.0 sec

NA: 8

LB: 0.0 PTS1d: 32768

WinNuts - \$(4-MeO2C)Ph4TOP_Pd 


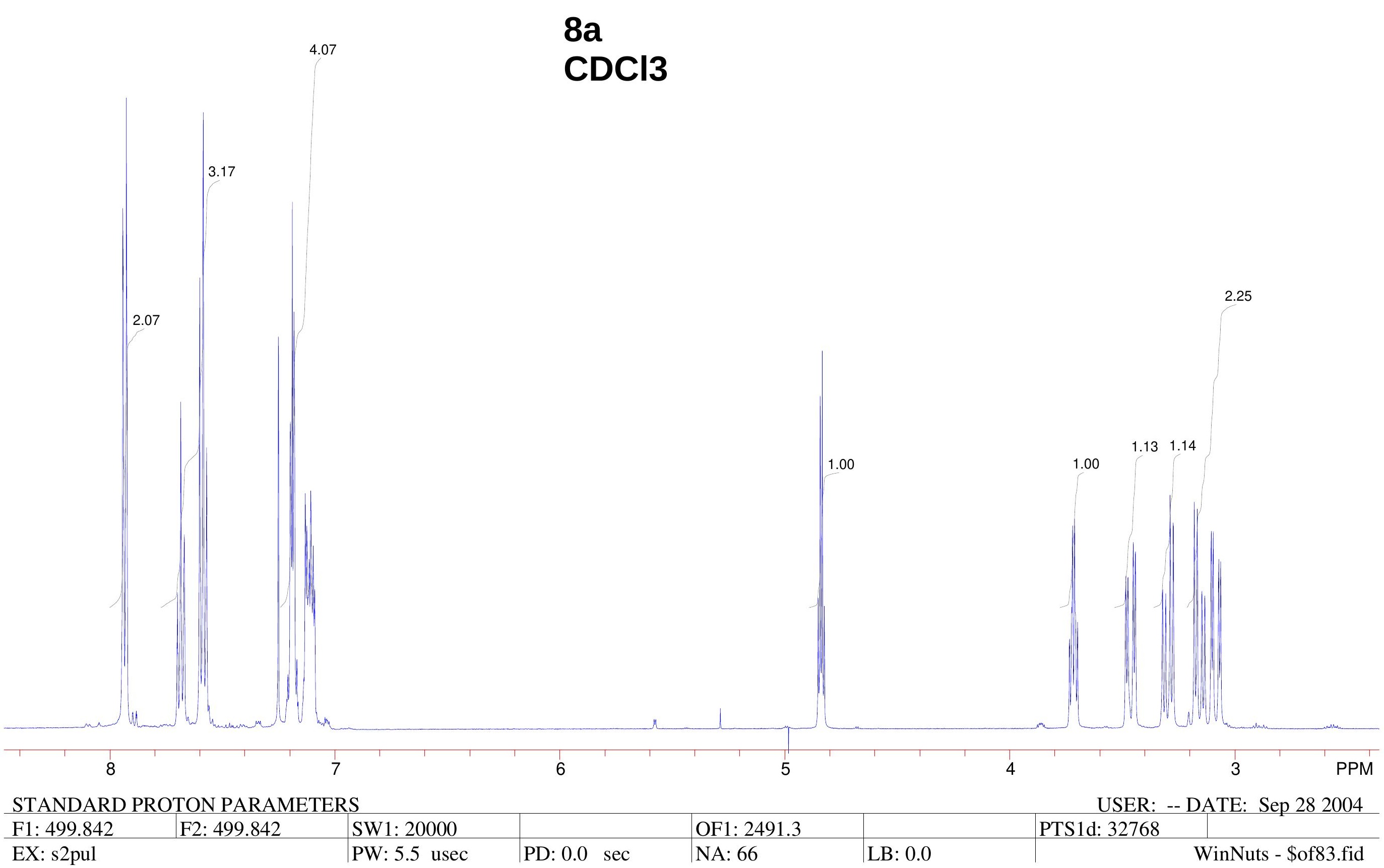


$8 a$

\section{$\mathrm{CDCl} 3$}

\section{6}

\begin{tabular}{c|c}
140 & 120 \\
STANDARD CARBON PARAMETERS
\end{tabular}

F1: 125.697

EX: s2pul
F2: 499.842

SW1: 25000

PW: 13.0 usec
100
80 PTS1d: 8192

OF1: 11940.4

NA: 2853

LB: 0.0

WinNuts - \$of83_13C.fid 
8b

CDCl3

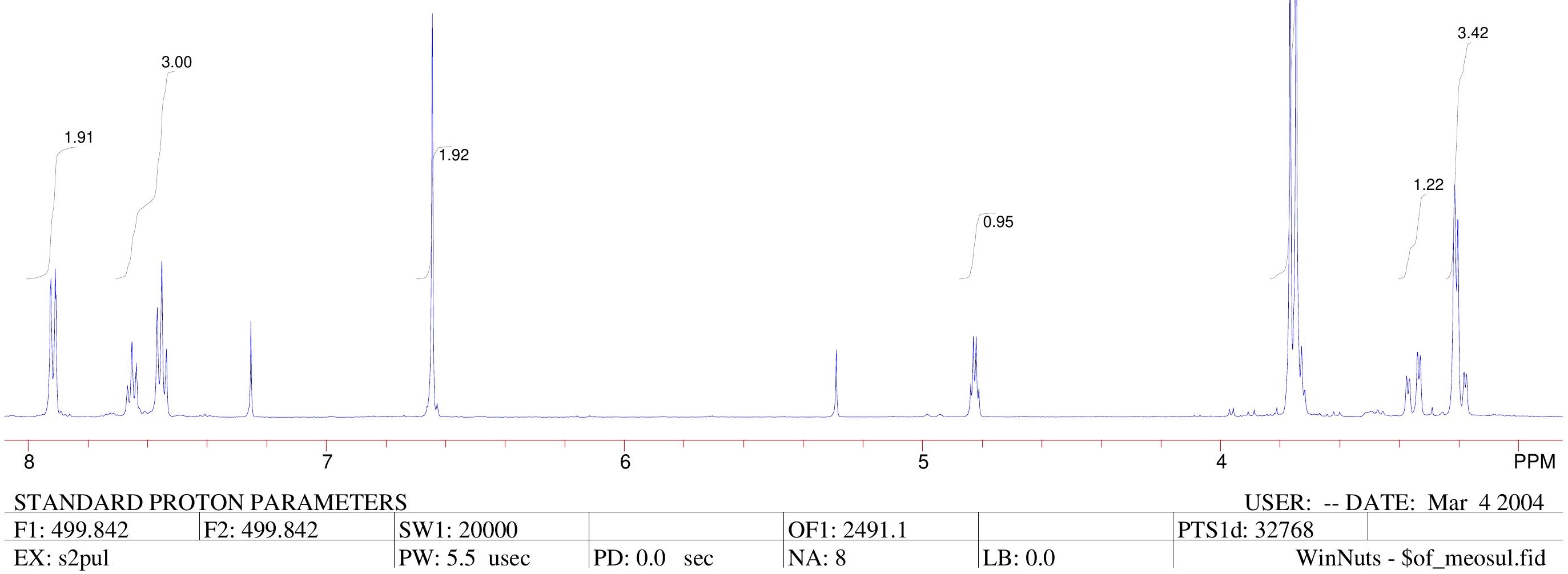

F1: 499.84

EX: s2pul
OF1:
NA: 8

LB: 0.0 


\section{$8 b$}

\section{C NMR in CDCl3}

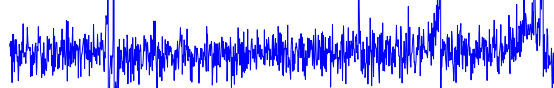

140

120

100

80

60

40

PPM

F1: 125.697 F2: 499.842

SW1: 25000

PW: 13.0 usec
OF1: 11937.4

NA: 1613

518

PD: $2.0 \mathrm{sec}$ PTS1d: 8192

WinNuts - \$of23_13C.fid 
 \\ $\mathrm{CDCl} 3$}

3.00

1.91

\begin{abstract}
F2: 499.855
\end{abstract}
SW1: 20000

PW: 3.0 usec
OF1: 2493.2

NA: 8
PTS1d: 32768

LB: 0.0

WinNuts - \$fb_3black.fid 


\section{CDCl3}
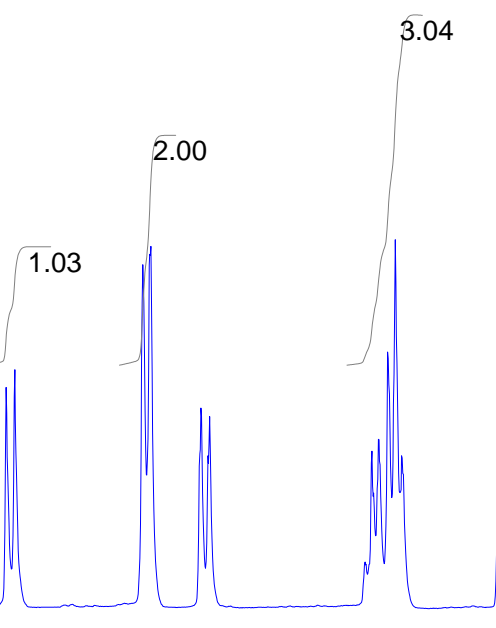

2.07

$$
8
$$

\section{6}

5

STANDARD PROTON PARAMETERS

F1: 499.855

F2: 499.855

SW1: 20000

PW: 5.5 usec

OF1: 2495.3

NA: 8 PTS1d: 32768

WinNuts - \$fb_1blue1.fid 


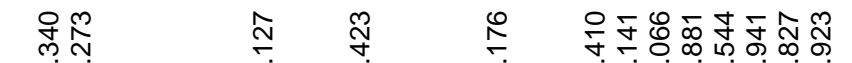

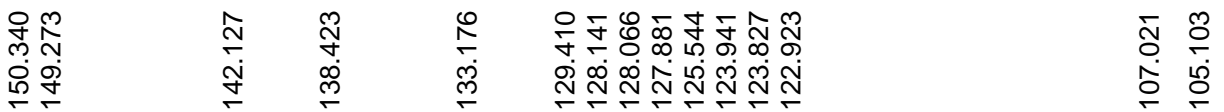

용్ㅇ

চ্口ে

으 용

8d

\section{CDCl3}

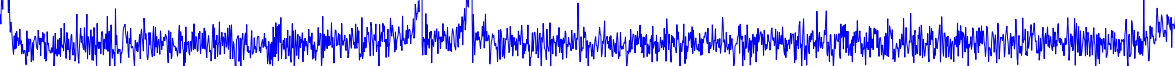

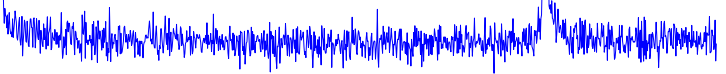

F1: 125.695 F2: 499.836

SW1: 25000

PW: 13.0 usec
OF1: 12175.4

NA: 1472

USER: -- DATE: Dec 92004 PTS1d: 8192

LB: 0.0

WinNuts - \$of_8d_13C.fid 

$\mathrm{CDCl} 3$

\begin{abstract}
F2: 499.842
\end{abstract}
SW1: 20000

PW: 5.5 usec

OF1: 2515.0

PD: $0.0 \mathrm{sec}$

NA: 8

LB: 0.0

USER: -- DATE: Oct 82004 PTS1d: 32768

WinNuts - \$of84_1H.fid 
9a CDCl3

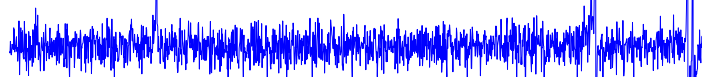

F1: 125.697

EX: s2pul

\begin{abstract}
F2: 499.842
\end{abstract}
SW1: 25000

PW: 13.0 usec
OF1: 11931.2 \begin{tabular}{|l|l|l|}
\hline PD: $2.0 \mathrm{sec}$ & $\mathrm{NA}: 933$ & LB: 0.0
\end{tabular}

USER: -- DATE: Oct 82004 PTS1d: 8192

WinNuts - \$of84_13C.fid 


\section{4}

\section{9b \\ CDCl3}

$$
\text { F2: } 499.855
$$

SW1: 20000

PW: 5.5 usec

OF1: 2493.5

NA: 8
PTS1d: 32768

LB: 0.0
3.00

PD: $0.0 \mathrm{sec}$

WinNuts - \$fb_meobz3.fid 


\section{9b $\mathrm{CDCl} 3$ 13C NMR - high field part}

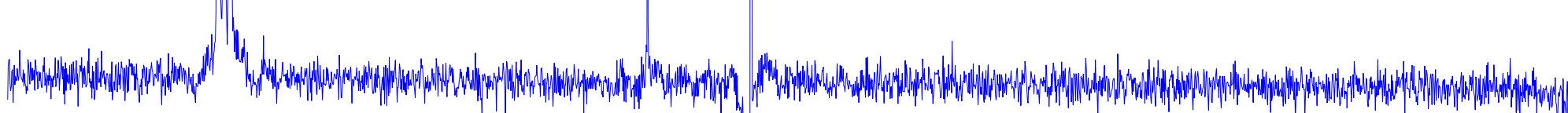

80 70

60

50

40

30

OF1: 11943.7

PD: $2.0 \mathrm{sec}$

NA: 16335

LB: 0.0 USER:

SW1: 25000 PW: 13.0 usec

F1: 125.700 F2: 499.855

1 198. $1000 \mathrm{~s}$

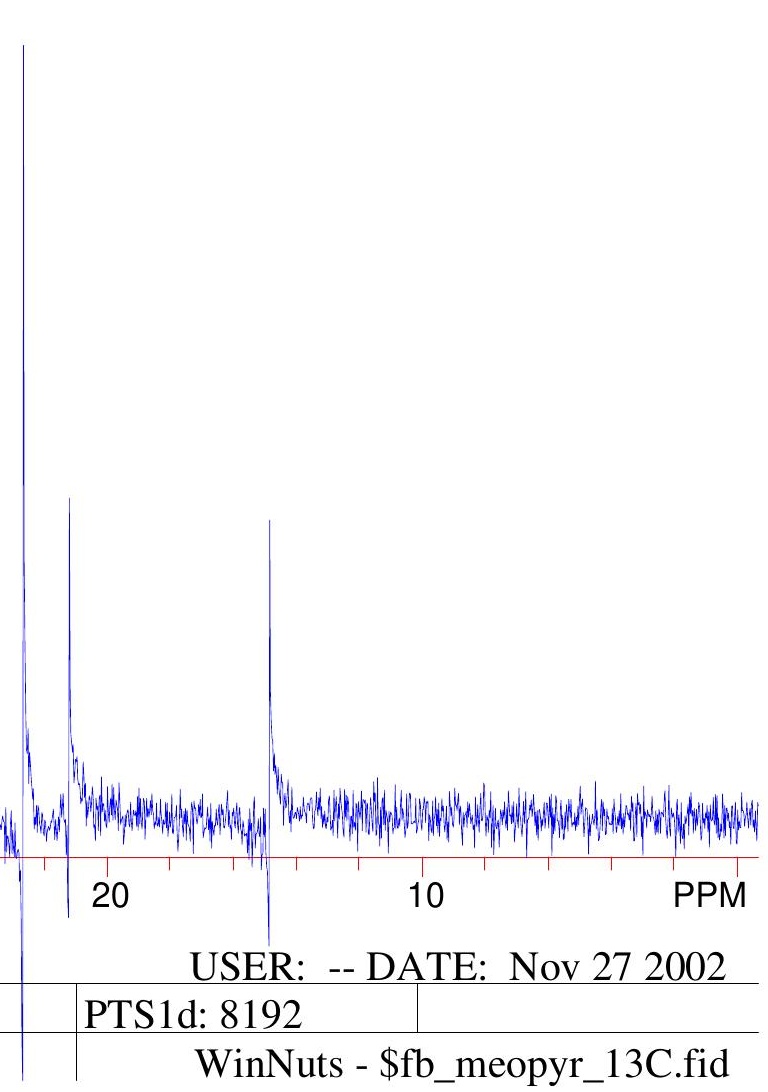




\section{9b}

CDCl3

13C NMR - low field part

F1: 125.700 F2: 499.855

SW1: 25000

PW: 13.0 usec

OF1: 11943.7

NA: 16335

LB: 0.0 PTS1d: 8192 


\section{$10 \mathrm{a}$}

$\mathrm{CDCl} 3$

4.00

\begin{abstract}
F2: 499.842
\end{abstract}
SW1: 20000

PW: 5.5 usec

OF1: 2479.2

PD: 0.0 sec

NA: 8

LB: 0.0 PTS1d: 32768

WinNuts - \$of86.fid 
$10 \mathrm{~b}$

$\mathrm{CDCl} 3$

$$
\text { F2: } 499.855
$$

SW1: 20000

PW: 5.5 usec
$6.5 \quad 6.0$ OF1: 2490.9

NA: 20

4.5

4.0 PPM

USER: -- DATE: Nov 302002 PTS1d: 32768

WinNuts - \$fb_meopyr_1H.fid 


\section{$10 b$ in $\mathrm{CDCl} 3$}

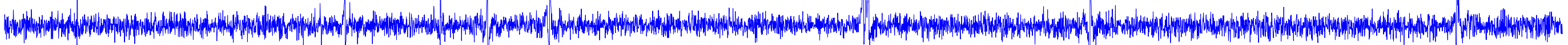

140

120

100

80

STANDARD CARBON PARAMETERS

F1: 125.700 F2: 499.855

SW1: 25000

PW: 13.0 usec
60

OF1: 11940.5

NA: 661

PD: 2.0 sec
40

20 PPM

USER: -- DATE: Nov 302002 PTS1d: 8192

WinNuts - \$fb_meopyrrol_13C.fid 


\section{4}

$\mathrm{CDCl} 3$

OF1: 2492.0

NA: 8
PD: 0.0 sec

\begin{abstract}
F2: 499.842
\end{abstract}
EX: s2pul 


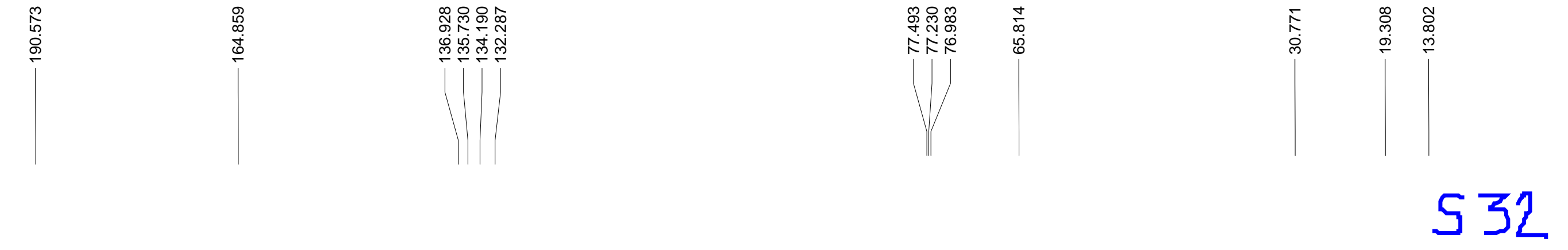

14 $\mathrm{CDCl} 3$

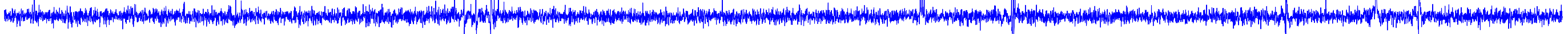

180

160

140

120

100

80

60

40

20

PPM

STANDARD CARBON PARAMETERS

F1: 125.697

F2: 499.842

SW1: 25000

PW: 13.0 usec

OF1: 11933.8

NA: 256

USER: -- DATE: Sep 242004 PTS1d: 8192

WinNuts - \$of32c_13C.fid 


$$
533
$$

\section{NMR spectra}

\section{2. $A r_{4}$ TNP's}


.24

\section{$12 \mathrm{a}$ - dication (* $2 \mathrm{CF} 3 \mathrm{COOH}$ ) in $\mathrm{CDCl} 3$}

SW1: 20000

PW: 5.5 usec

NA: 8

USER: -- DATE: Oct 152004 PTS1d: 32768

WinNuts - \$of87dc.fid 


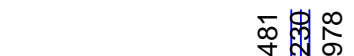

\section{2a - dication (* 2 CF3COOH)} in $\mathrm{CDCl} 3$ PTS1d: 8192

F1: 125.697 EX: s2pul

\begin{abstract}
F2: 499.842
\end{abstract}
SW1: 25000

PW: 13.0 usec
OF1: 11940.6

NA: 17408

LB: 0.0

WinNuts - \$of87dc_13C.fid 


\section{$12 \mathrm{~b}$ - dication ( $* 2 \mathrm{CF} 3 \mathrm{COOH})$ in $\mathrm{CDCl} 3(\mathrm{~T}=310 \mathrm{~K})$}

USER: shash -- DATE: Mon Apr 19 07:48:04 2004 


\section{$12 \mathrm{~b}$ - dication ( * $2 \mathrm{CF} 3 \mathrm{COOH}$ ) in $\mathrm{CDCl} 3(\mathrm{~T}=310 \mathrm{~K})$}

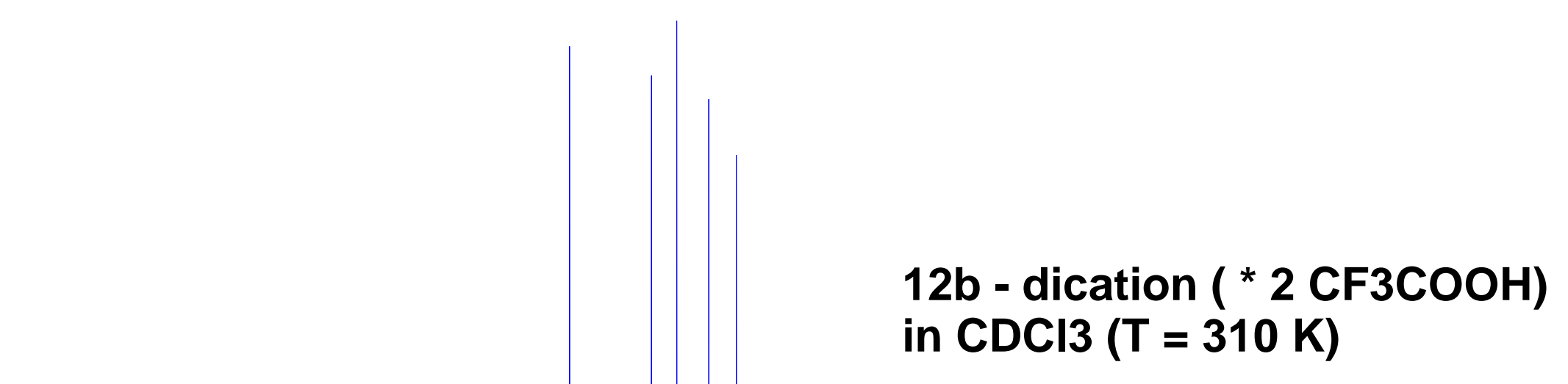

USER: gosha -- DATE: Mon Apr 19 08:27:29 2004 


\section{2e - dication ( * 2 CF3COOH)} in $\mathrm{CDCl} 3$

\section{9}

OF1: 2488.4

PD: 0.0 sec

\begin{abstract}
F2: 499.842
\end{abstract} $-2$
USER: -- DATE: Oct 162004 PTS1d: 32768

WinNuts - \$of88dc.fid 


\section{2e - dication ( * 2 CF3COOH) in $\mathrm{CDCl} 3$}

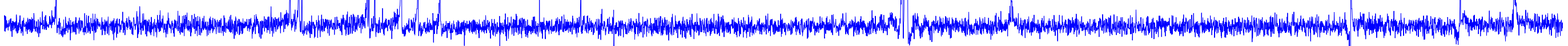

F1: 125.697

EX: s2pul

\begin{abstract}
F2: 499.842
\end{abstract}
SW1: 25000

PW: 13.0 usec
OF1: 11941.1

PD: 2.0 sec

NA: 8740
USER: -- DATE: Oct 162004 PTS1d: 8192

WinNuts - \$of88_13c.fid 


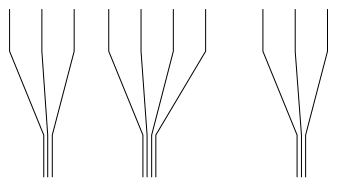

\title{
541
}

\section{$12 \mathrm{f}$ in $\mathrm{CDCl} 3$}

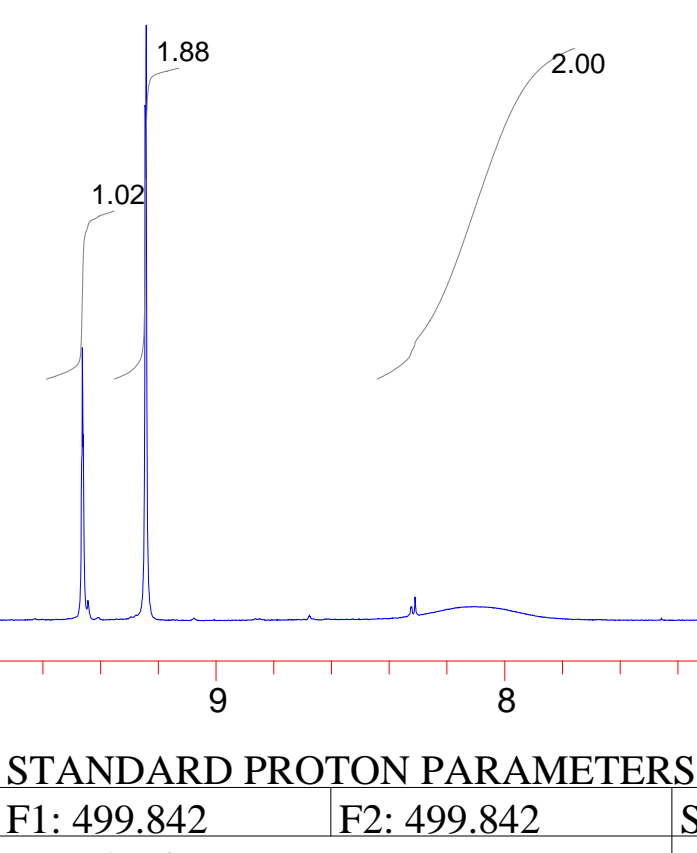

F1: 499.842

EX: s2pul

\begin{abstract}
F2: 499.842
\end{abstract}
SW1: 20000

PW: 5.5 usec

2.11

\begin{abstract}
4
\end{abstract}
3

2

PPM USER: -- DATE: Apr 52004 OF1: 2491.7 NA: 8 PTS1d: 32768 


\section{$12 f$ in pyridine-d5}

13C NMR, high field region

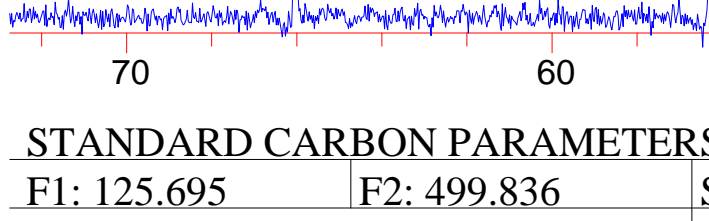

F1: 125.695

EX: s2pul

\begin{abstract}
F2: 499.836
\end{abstract}
SW1: 25000

PW: 13.0 usec
50

(n)

30

20 USER: -- DATE: Dec 102004 PTS1d: 8192

OF1: 12176.7

NA: 15472
PD: 2.0 sec 


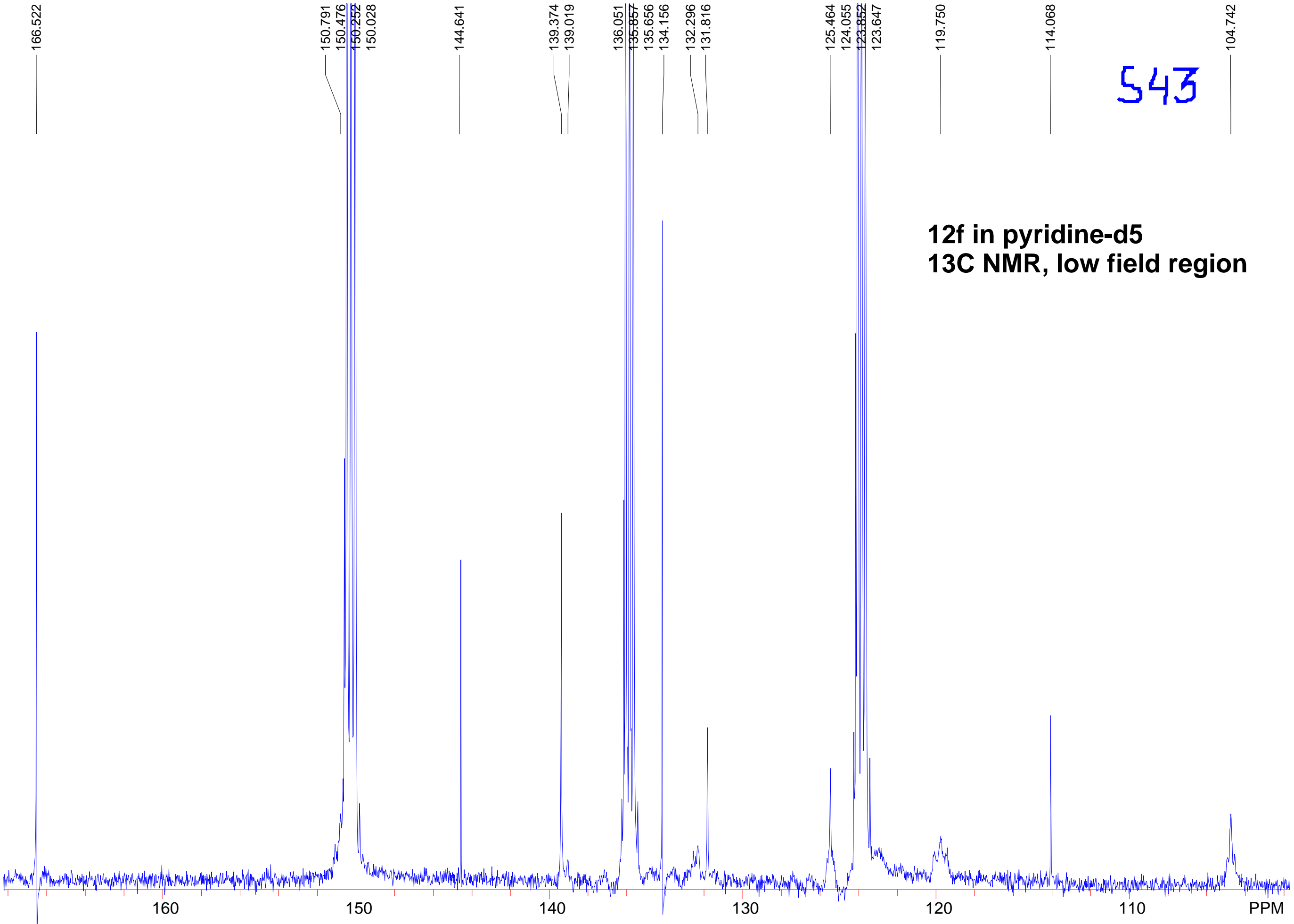
F2: 499.836

USER: -- DATE: Dec 102004 EX: s2pul

OF1: 12176.7

NA: 15472 PTS1d: 8192

LB: 0.0

WinNuts - \$of8h_13C.fid 


\section{Pd-12b in CDCl3-pyridine-d5}

water

9

8

7

6

5

4

PPM STANDARD PROTON PARAMETERS F1: 499.855 F2: 499.855

OF1: 2657.5

PD: 0.0 sec

NA: 100

USER: -- DATE: Dec 102002 PTS1d: 32768

WinNuts - \$(4-MeO2C)Ph4TNP_Pd_py 


\section{Pd-12b in CDCl3-pyridine-d5}

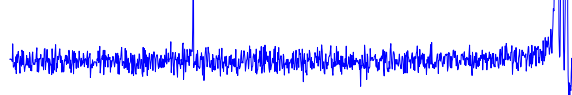

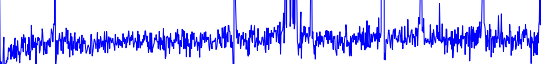

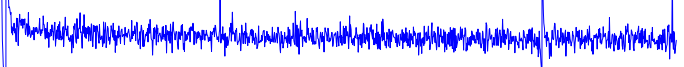

F1: 125.700 F2: 499.855

SW1: 25000

PW: 13.0 usec

OF1: 11965.3

PD: $2.0 \mathrm{sec}$

NA: 14607

LB: 0.0 PTS1d: 8192

USER: -- DATE: Dec 152002 WinNuts - \$(4-MeO2C)Ph4TNPpd_13C.fid 


\section{$\mathrm{Pd}-12 \mathrm{~b}$ in $\mathrm{CDCl}$ 3-pyridine-d5}


Pd-12d in PhNO2-d5 - dmso-d6 (T = $80 \stackrel{\circ}{\mathrm{C}})$

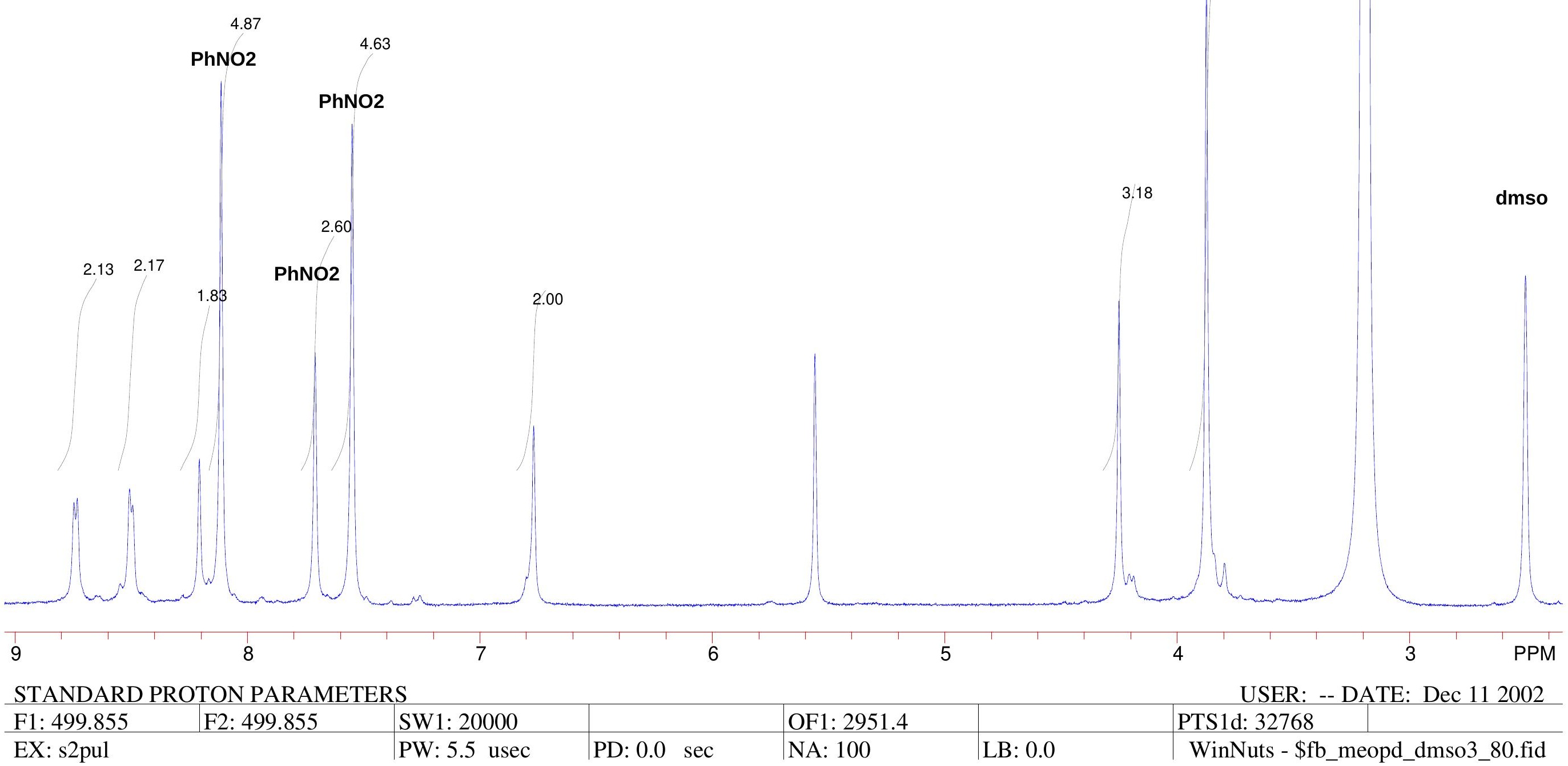




\section{Pd-12e in pyridine-d5}

py

py

F2: 499.842

SW1: 20000

PW: 5.5 usec

OF1: 2506.2

PD: 0.0 sec

NA: 8

LB: 0.0

USER: -- DATE: Oct 202004 PTS1d: 32768 


\section{Pd-12f in pyridine-d5 - enlarged}

STANDARD CARBON PARAMETERS

F1: 125.695 EX: s2pul F2: 499.836

SW1: 25000 PW: 13.0 usec
$148 \quad 146$

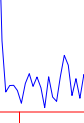

144
138 USER: OF1: 12188.2 NA: 3968
140

-- DATE: Nov 182004 WinNuts - \$of91_13C.fid 
Zn-12c in pyridine-d5 ( $T=80 \stackrel{\circ}{\mathrm{C}})$

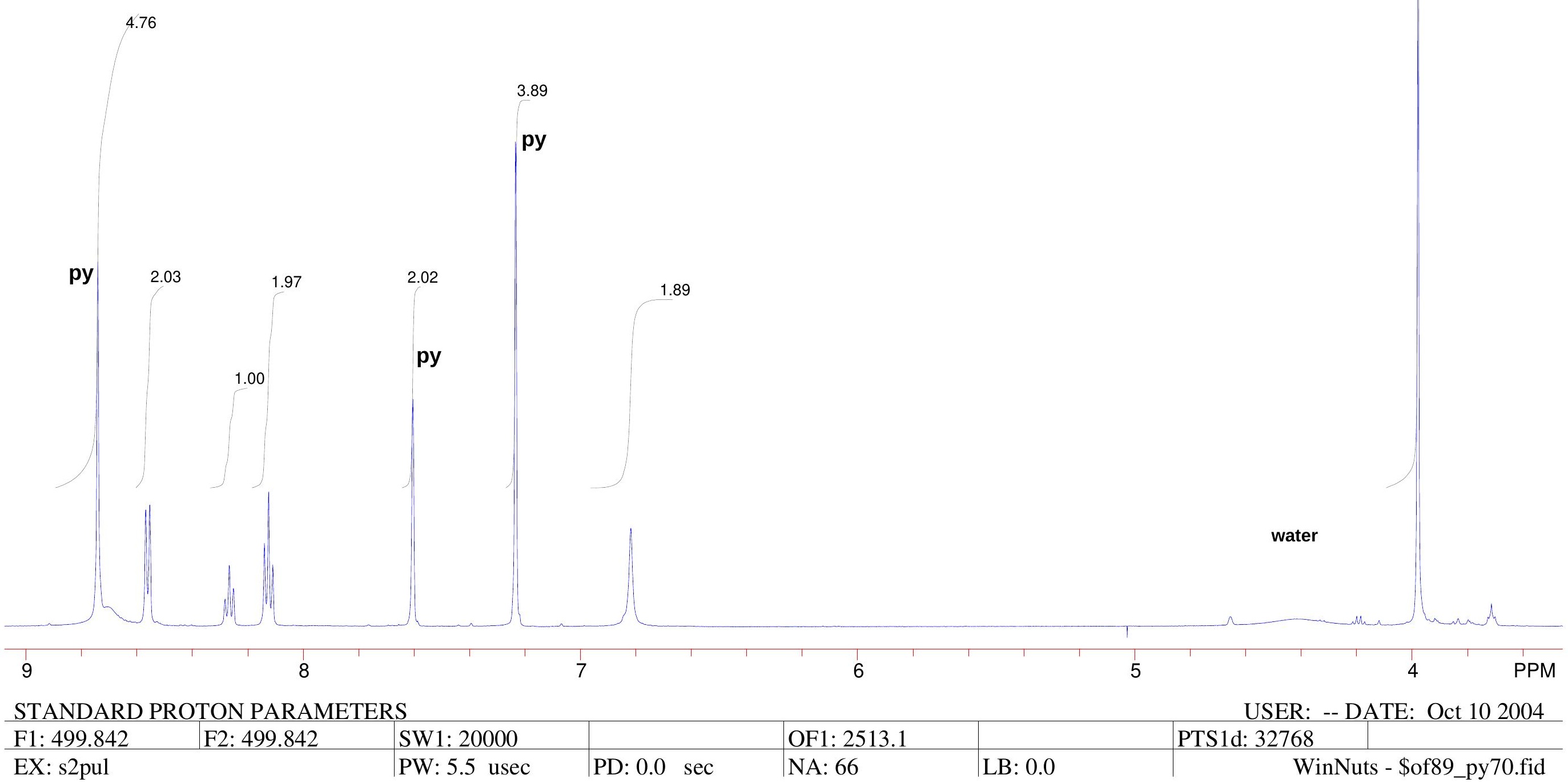




\section{$\mathrm{Zn-12d}$ in pyridine-d5}

NA: 8 


\section{6}

\section{$\mathrm{Zn-12d}$ in pyridine-d5 ( $T=50 \mathrm{C})$}

F1: 125.695 F2: 499.836

SW1: 25000

PW: 13.0 usec
OF1: 12190.3

NA: 3271 PTS1d: 8192

WinNuts - \$of89_13C.fid 